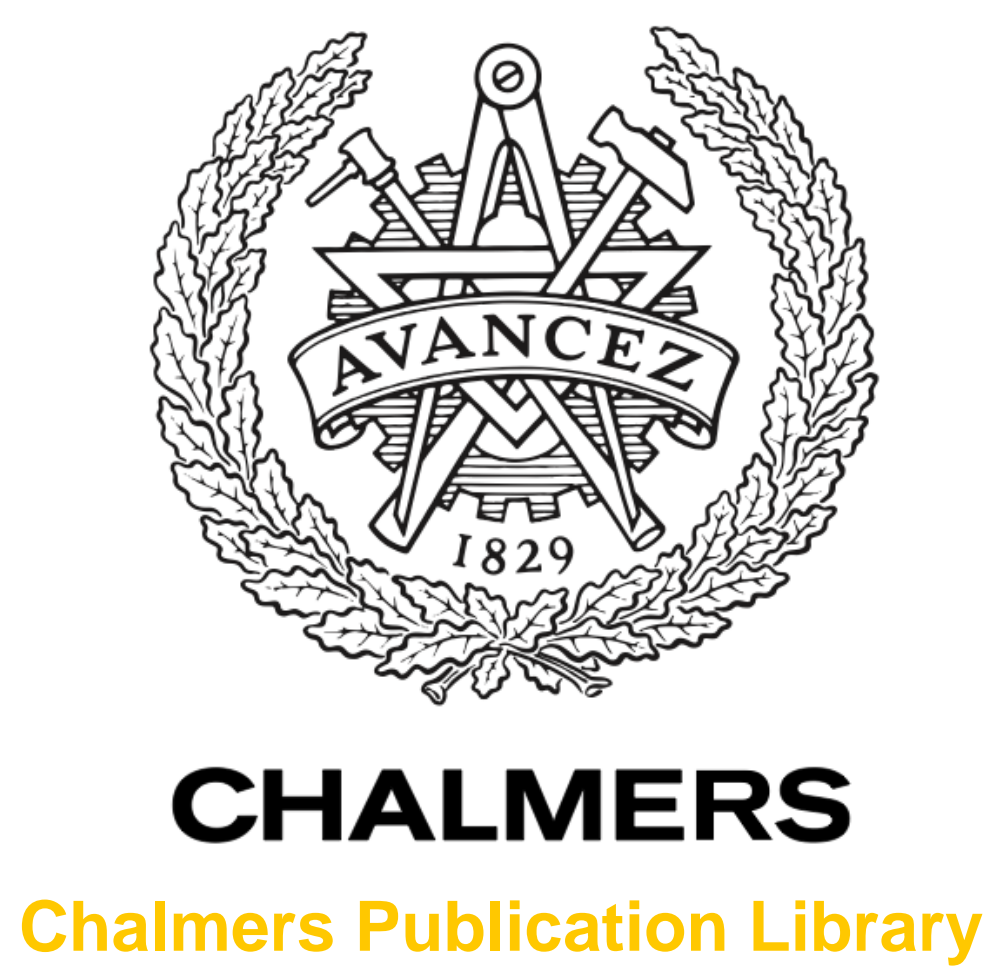

HARQ in Poisson Point Process-based Heterogeneous Networks

This document has been downloaded from Chalmers Publication Library (CPL). It is the author's version of a work that was accepted for publication in:

IEEE Vehicular Technology Conference (ISSN: 15502252)

Citation for the published paper:

Fang, C. ; Makki, B. ; Svensson, T. et al. (2015) "HARQ in Poisson Point Process-based Heterogeneous Networks". IEEE Vehicular Technology Conference, vol. 2015

http://dx.doi.org/10.1109/VTCSpring.2015.7145855

Downloaded from: http://publications.lib.chalmers.se/publication/204247

Notice: Changes introduced as a result of publishing processes such as copy-editing and formatting may not be reflected in this document. For a definitive version of this work, please refer to the published source. Please note that access to the published version might require a subscription.

Chalmers Publication Library (CPL) offers the possibility of retrieving research publications produced at Chalmers University of Technology. It covers all types of publications: articles, dissertations, licentiate theses, masters theses, conference papers, reports etc. Since 2006 it is the official tool for Chalmers official publication statistics. To ensure that Chalmers research results are disseminated as widely as possible, an Open Access Policy has been adopted.

The CPL service is administrated and maintained by Chalmers Library. 


\title{
HARQ in Poisson Point Process-based Heterogeneous Networks
}

\author{
Chao Fang*, Behrooz Makki*, Yateng Hong ${ }^{\dagger}$, Xiaodong Xu ${ }^{\dagger}$, Tommy Svensson * \\ *Department of Signals and Systems, Chalmers University of Technology, Gothenburg, Sweden \\ fchao@student.chalmers.se, \{behrooz.makki, tommy.svensson\}@chalmers.se \\ ${ }^{\dagger}$ Beijing University of Posts and Telecommunications, Beijing, China \\ \{toothache, xuxiaodong\}@bupt.edu.cn
}

\begin{abstract}
Hybrid automatic repeat request (HARQ) plays an important role in improving the transmission efficiency and the robustness of wireless networks. Considering $K$-tier heterogeneous networks (HetNets) and modelling the locations of the base stations (BSs) as a homogeneous Poisson point process (PPP), this paper investigates the performance of HetNets implementing HARQ. We give closed-form expressions for the quality of service $(\mathrm{QoS})$ coverage probability which is defined in terms of whether the received signal quality is above a predetermined threshold, and the per-user throughput with HARQ. We show that using HARQ can indeed improve the QoS coverage probability. However, depending on the channel conditions, the per-user throughput of the HetNets may decrease by the implementation of HARQ. Furthermore, we show that the small cell density has negligible effect on the QoS coverage probability and the peruser throughput, and the per-user throughput may increase with the small cell path loss.
\end{abstract}

\section{INTRODUCTION}

The key challenge for the next generation wireless network is the large growth in data traffic, connected devices and various performance requirements. One solution is to develop heterogeneous cellular networks (HetNets) by deploying different tiers of base stations (BSs) [1]. The idea is to use the tier- 1 cells to provide the highest transmitting power and the largest coverage area along with low-power and lowcoverage small cells to reduce outage and improve the hotspot throughput inside tier-1 cells, e.g., the 3-tier cellular networks consist of macro cells, pico cells and femto cells with descending transmit power [1]-[3]. However, this type of network topology poses new challenges to the network performance analysis. Due to the random distribution of the small cells and the lack of dedicated planning, the conventional hexagonal grid model and the Wyner model [4] are no longer suitable for modelling the randomly distributed $K$-tire of networks. In order to capture the random node distribution in HetNets, [5], [6] suggests a tractable and well-known model by using the tools from stochastic geometry.

Modelling the locations of the BSs as a Poisson point process (PPP), many researchers study the quality of service (QoS) coverage probability which measures the probability that the received signal-to-interference-plus-noise ratio (SINR) is above a predetermined threshold. Closed-form expressions for the QoS coverage probability are derived for a single tier network [7] and are further extended into $K$-tier HetNets [8], [9]. In the HetNets environment, it is preferred to connect a user to a lightly-loaded small cell rather than a heavilyloaded large cell. Therefore, considering the cell association strategy, [9] introduces a bias factor to weight the received signal power, thus achieving better load-balancing. Although the PPP may not be appropriate to model large cells between which the distance is usually large, [7] shows that the PPP model is almost as accurate as the grid model in a single tier cellular network.

Due to the limited power and high interference, many technologies have been applied to achieve the coverage requirements in the $K$-tier networks. Based on the PPP model, some of the leading techniques, such as inter-cell interference coordination [10] and multi-antenna transmission [11] have been studied by numerous researchers. However, little research has been conducted into analyzing the hybrid automatic repeat request (HARQ) in the PPP-based HetNets. On the other hand, considering single-user setups, many works, e.g., [12]-[14], have previously showed that HARQ can effectively reduce the probability of outage and increase the throughput in wireless networks. For this reason, it is important to study the benefits of HARQ in HetNets, in order to design efficient systems. The problem becomes more important when we remember that the HARQ protocols have been already provided in many wireless standards, e.g., IEEE 802.11n, IEEE 802.16e and LTE-A [15]-[17]. Hence, needing no additional design, HARQ becomes a cost- and complexity-efficient approach to fulfil different quality-of-service requirements. In addition, HARQ is an efficient sequential feedback approach to provide the BSs with partial channel state information (CSI), while other partial CSI feedback schemes (such as CSI quantisation) are usually difficult to implement in large HetNets. Therefore, HARQ not only presents potentials for performance improvements, but also is an effective and realistic approach in HetNets.

This paper incorporates the HARQ into the PPP-based HetNet model and study some of the key network metrics. First, we build a system model for the $K$-tier HetNet based on the PPP model and introduce HARQ by retransmitting the data under certain conditions. Next, considering PPP-based HetNets with different BS density parameters, we derive closed-form expressions for the long-term throughput and the QoS coverage probability, and analyze the system performance in the cases using repetition time diversity (RTD) HARQ protocols. Finally, using the closed-form expressions, we evaluate the 
effect of different parameters such as the BS density, the codewords rates and the small cell path loss on the system performance.

The results show that HARQ can effectively increase the QoS coverage probability. Particularly, the highest performance gains are achieved with few numbers of HARQ-based retransmissions, while, the relative gain in the QoS coverage probability decreases as the maximum number of permitted retransmissions increases. On the other hand, depending on the channel condition, the implementation of HARQ protocols may decrease the per-user throughput of the HetNets. This is in contrast to the results in, e.g. [12]-[14], where they consider the single-transmitter setup and the throughput is shown to increase via HARQ-based data transmission. We show that adding more small cells into the network does not affect the QoS coverage probability and the per-user throughput significantly, but provides much potential to reduce the outage zones in large cells and increase the overall throughput.

\section{Downlink System Model}

In $K$-tier HetNet, the BSs from different tiers usually vary in transmit power, spatial density and coverage area. We assume the BSs in the $i$-th tier, $1 \leq i \leq K$, are spatially distributed according to a 2D homogeneous PPP $\Phi_{i}$ with density $\lambda_{i}$, and transmit with constant power $P_{i}$. Without loss of generality, we consider the downlink transmission to a user placed at the origin of the plane.

As one of the most efficient HARQ protocols, we consider the RTD HARQ, while the results can be easily extended to the cases with, e.g., incremental redundancy HARQ. The use of RTD HARQ is of particular interest in large-scale HetNets because it implies low implementation complexity. We assume no instantaneous CSI at the BSs and perfect CSI at the user. In this way, in each packet transmission, every BS sends the data with the initial rate $R$. If the instantaneous SINR at the receiver supports the rate, i.e., $\log (1+\mathrm{SINR})>R$, the transmission is successful and the message is correctly decoded. Otherwise, the BS retransmits the same data until the message is correctly decoded or the number of transmissions exceeds a permitted maximum number $M+1$. The receiver jointly decodes received signals using maximum ratio combining and, depending on the message decoding status, sends acknowledgement/negative acknowledgement (ACK/NACK) feedback bits after each reception. The feedback channel bits are assumed to be received by the BSs error-free.

Considering block fading channels, the signal sent from an arbitrary BS $x_{i}$ in the $i$-th tier is subject to a path loss $\left\|x_{i}\right\|^{-\alpha_{i}}$ and Rayleigh fading with unit mean, where $\alpha_{i}>2$ is the path loss exponent. For simplicity, we assume that the uncoordinated frequency reuse scheme with reuse factor 1 is used and the thermal noise is negligible compared to the interference power caused by BSs, therefore we consider the network as interference limited.

For cell association strategies, we assume the user is associated to the BS providing the maximum long-term averaged power. The system is fully loaded and all BSs except the associated one cause interference to the considered user. No handovers occur until all the (re)transmissions end. The performance is analyzed in quasi-static conditions where the packet time $^{1}$ is less than a single block fading duration, which is reasonable for stationary or slowly moving users, since each transmission time is much less than the channel coherence time according to the LTE standard, as discussed in [18], [19].

If the user is associated to the $i$-th tier and is at a random distance $\left\|x_{i}\right\|$ from its associated $\mathrm{BS}$, the received signal-tointerference ratio (SIR) for each transmission is given by

$$
\operatorname{SIR}_{i}=\frac{P_{i} h_{x_{i}}\left\|x_{i}\right\|^{-\alpha_{i}}}{\sum_{j=1}^{K} \sum_{x_{j} \in \Phi_{j} \backslash x_{i}} P_{j}\left\|x_{j}\right\|^{-\alpha_{j} h_{x_{j}}}} .
$$

where $h_{x}$ is the independent and identically distributed fading coefficients and follow the exponential distribution with unit mean.

In Sections III and IV, we use the system model to evaluate the effect of the HARQ protocols on the QoS coverage probability and the throughput of the PPP-based HetNets, respectively.

\section{QoS Coverage Probability}

In this section, we calculate the QoS coverage probability in $K$-tier HetNet using HARQ. Since each user can be associated to only one tier at the same time and does not change the associated BS during the retransmissions, we first give the expression of the QoS coverage probability for each tier and then extend it to be averaged over all tiers.

Assuming that the user is associated to a BS in the $i$-th tier and uses the maximum ratio combining of the received signals, the combined SIR after $m$-th (re)transmission is $m \times \mathrm{SIR}_{i}$, where $0<m \leq M+1$, and the resulting data rate decreases to $R / m$ after $m$ (re)transmissions. In this case, a user is covered and the message is correctly decoded, if the combined SIR at the end of the $m$-th (re)transmission supports the data rate (and not before). Hence, the QoS coverage probability conditioned on that the user is associated to the $i$-th tier is found as

$$
\begin{aligned}
p_{i}= & \sum_{m=1}^{M+1} \operatorname{Pr}\left(\log \left(1+(m-1) \operatorname{SIR}_{i}\right)<R\right. \\
& \left.\leq \log \left(1+m \operatorname{SIR}_{i}\right)\right) \\
= & \operatorname{Pr}\left(\operatorname{SIR}_{i}>\frac{e^{R}-1}{M+1}\right) .
\end{aligned}
$$

Here, (2) is based on the fact that with the instantaneous SIR of $\mathrm{SIR}_{i}$, the maximum decodable rate is $\frac{1}{m} \log \left(1+m \mathrm{SIR}_{i}\right)$, if the same codeword is transmitted $m$ times and maximum ratio combining is utilised by the receiver.

Denoting the probability that a user is connected to an $i$ th tier BS by $a_{i}$, [9] gives the explicit expression for the cell association probability. As will be seen later, the cell association probability is cancelled out in the final expression

\footnotetext{
${ }^{1}$ A packet is defined as the transmission of a codeword along with all its possible retransmissions.
} 
of the overall QoS coverage probability, therefore we do not study $a_{i}$ in detail and denote the QoS coverage probability averaged over $K$ tiers as

$$
p_{c}=\sum_{i=1}^{K} a_{i} p_{i} .
$$

The cumulative distribution function of the SINR, i.e. SIR in our interference limited case, conditioned on the user associated to the $i$-th tier is derived as [9, Eq. 15]

$$
\operatorname{Pr}\left(\operatorname{SIR}_{i}<x\right)=1-\frac{2 \pi \lambda_{i}}{a_{i}} \int_{0}^{\infty} r e^{-\frac{x}{\operatorname{SNR}}-\pi \sum_{j=1}^{K} C_{j} r^{2 \alpha_{i} / \alpha_{j}}} \mathrm{~d} r
$$

where SNR $=\frac{P_{i} r^{\alpha_{i}}}{N_{0}}, N_{0}$ is the additive noise power and

$$
\begin{aligned}
& C_{j}= \lambda_{j} \frac{P_{j}}{P_{i}}\left[{\frac{B_{j}}{B_{i}}}^{2 / \alpha_{j}}+\frac{2 x\left(B_{j} / B_{i}\right)^{2 / \alpha_{j}-1}}{\alpha_{j}-2} \times\right. \\
&\left.{ }_{2} F_{1}\left[1,1-\frac{2}{\alpha_{j}} ; 2-\frac{2}{\alpha_{j}} ;-\frac{x B_{i}}{B_{j}}\right]\right],
\end{aligned}
$$

with $B_{i}$ denoting the bias given to the average received power from $i$-th tier BSs, and ${ }_{2} F_{1}[\cdot]$ being the Gauss hypergeometric function.

In this paper, we concentrate on the effects of the HARQ. Thus we simplify by setting all bias factors $B_{i}$ equal to 1 and considering the interference-limited scenario, we rewrite $[9$, Eq. 15] as

$$
\operatorname{Pr}\left(\operatorname{SIR}_{i}<x\right)=1-\frac{2 \pi \lambda_{i}}{a_{i}} \int_{0}^{\infty} r e^{-\pi \sum_{j=1}^{K} C_{j}(x) r^{2 \alpha_{i} / \alpha_{j}}} \mathrm{~d} r,
$$

where

$C_{j}(x)=\lambda_{j}{\frac{P_{j}}{P_{i}}}^{\frac{2}{\alpha_{j}}}\left(1+\frac{2 x}{\alpha_{j}-2}{ }^{2} F_{1}\left[1,1-\frac{2}{\alpha_{j}} ; 2-\frac{2}{\alpha_{j}} ;-x\right]\right)$

Combining (4), (7) and (8), the overall QoS coverage probability of a user in a $K$-tier heterogenous network with HARQ as a function of $M, R$ is given by

$$
\begin{aligned}
p_{c}(M+1, R) & =\operatorname{Pr}\left(\operatorname{SIR}>\frac{e^{R}-1}{M+1}\right) \\
& =\sum_{i=1}^{K} 2 \pi \lambda_{i} \int_{0}^{\infty} r e^{-\pi \sum_{j=1}^{K} C_{j}\left(\frac{e^{R}-1}{M+1}\right) r^{2 \alpha_{i} / \alpha_{j}}} \mathrm{~d} r .
\end{aligned}
$$

Figure 1 shows the effects of the HARQ on the QoS coverage probability. (Note that $M=0$ gives the QoS coverage probability without HARQ in [9]). As expected, the larger the maximum number of retransmissions, the better the QoS coverage probability. Moreover, we observe that the most gains of the HARQ are achieved with low values of $M$. I.e. the relative gain in the QoS coverage probability decreases with $M$, the largest gain occurs when from no HARQ to using HARQ with $M=1$. In the meantime, using (9), it

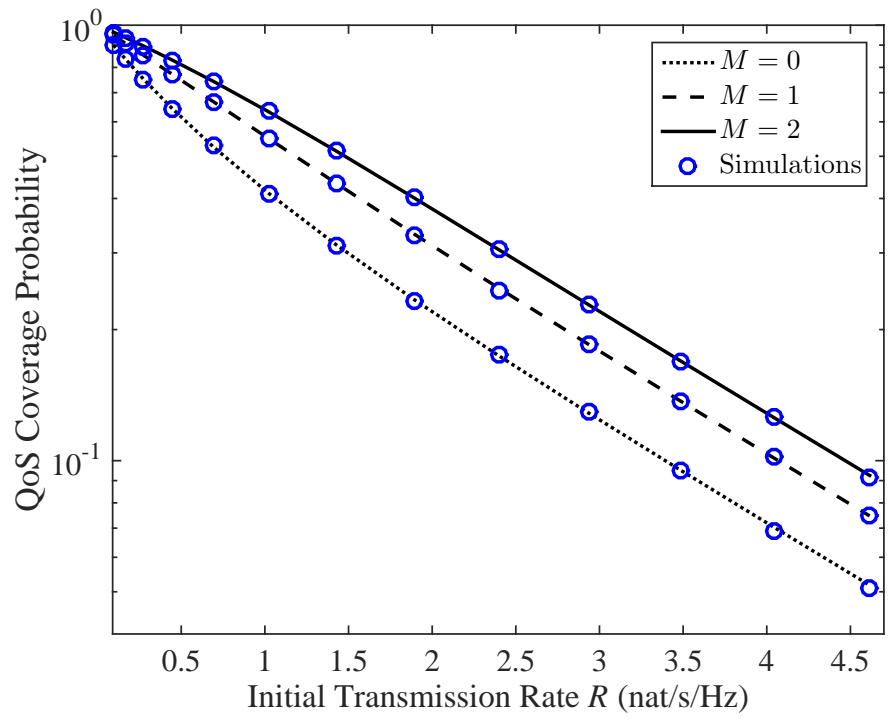

Figure 1. QoS Coverage probability under HARQ for $M=0,1,2$ in a 3 -tier HetNet. For tier-1 BSs, the path loss exponent, the BS density and the transmit power are set to $\alpha_{1}=3.8, \lambda_{1}=\frac{1}{500^{2}}$ and $P_{1}=53 \mathrm{dBm}$, respectively. For tier-2 BSs, these parameters are $\alpha_{2}=3.5, \lambda_{2}=2 \lambda_{1}$ and $P_{2}=33 \mathrm{dBm}$, respectively. For tier-3 BSs, these parameters are $\alpha_{3}=4$, $\lambda_{3}=20 \lambda_{1}$ and $P_{3}=23 \mathrm{dBm}$, respectively. The simulations use an additive noise with power $-60 \mathrm{dBm}$, the comparison with the analytical results in the interference-limited case indicates the negligible effect of the additive noise in dense networks.

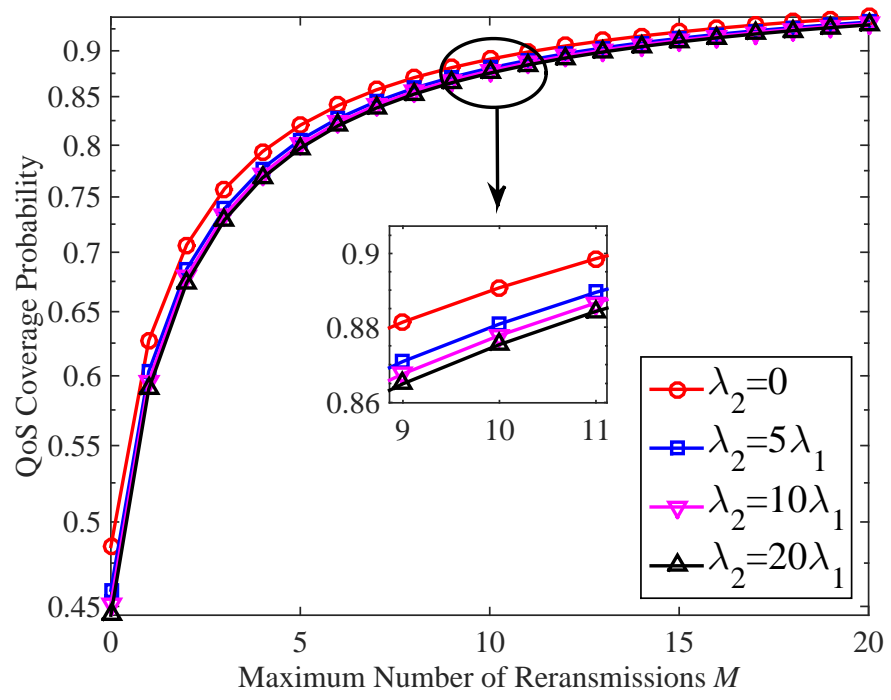

Figure 2. QoS Coverage probability versus the maximum number of retransmissions $M$ in a 2-tier HetNet. The initial transmission rate of all BSs is set to $R=0.8149 \mathrm{nat} / \mathrm{s} / \mathrm{Hz}$. For tier-1 BSs, the path loss exponent, the BS density and the transmit power are set to $\alpha_{1}=3.8, \lambda_{1}=\frac{1}{\pi 500^{2}}$ and $P_{1}=53 \mathrm{dBm}$, respectively. For tier- $2 \mathrm{BSs}$, these parameters are considered as $\alpha_{2}=3.5$, $\lambda_{2}=0,5 \lambda_{1}, 10 \lambda_{1}, 20 \lambda_{1}$ and $P_{2}=33 \mathrm{dBm}$, respectively.

is straightforward to show that $p_{c} \rightarrow 1$ if $M \rightarrow \infty, \forall R<\infty$. In Figure 2, we calculate the QoS coverage probability for different values of the maximum number of retransmissions and the second tier BS density. The QoS coverage improves with $M$ for all $\lambda_{2}$, and the increment is small for large $M$. Also, we observe that, in this setup, the QoS coverage is 


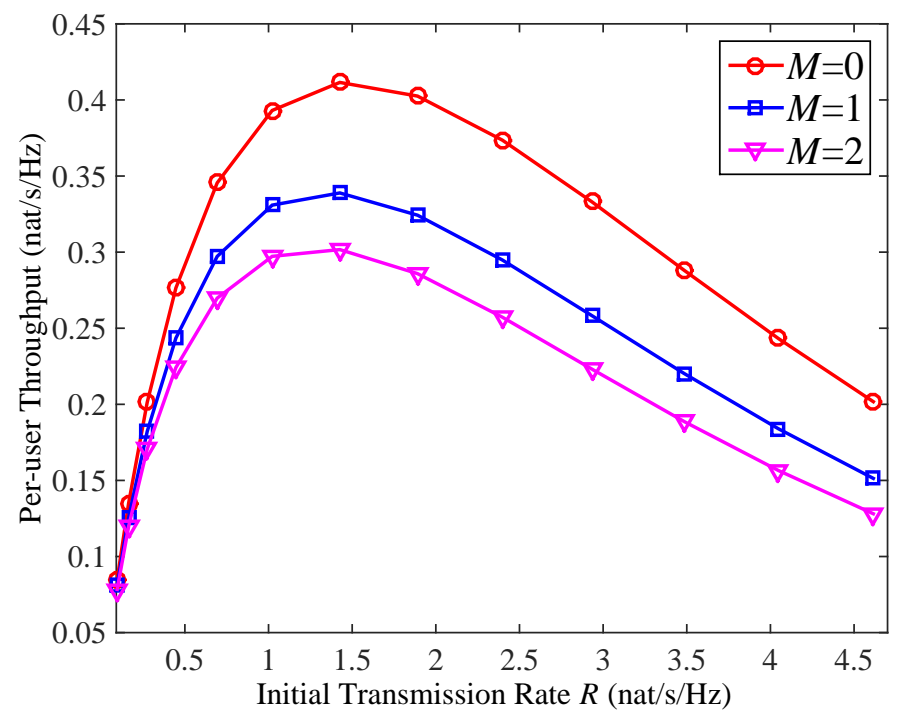

Figure 3. Per-user throughput under HARQ for $M=0,1,2$ in a 2- tier HetNet. The path loss exponent, the BS density and the transmit power of tier 1 are set to $\alpha_{1}=3.8, \lambda_{1}=\frac{1}{\pi 500^{2}}$ and $P_{1}=53 \mathrm{dBm}$, respectively. For tier 2, these parameters are considered as $\alpha_{2}=3.5, \lambda_{2}=10 \lambda_{1}$ and $P_{2}=33$ $\mathrm{dBm}$, respectively.

deteriorated when we first introduce the small cells. However, further increasing the density of the second tier BSs has little effect on the QoS coverage probability. The results imply that we can increase the number of small cells to reduce the outage zones inside tier-1 cells without decreasing the overall QoS coverage probability significantly.

\section{Per-user Throughrut}

In this section, we study the per-user throughput of a $K$-tier HetNet in the presence of HARQ. Assuming only one active user per cell, the per-user throughput measures the successful data rate over a long period with many packet transmissions. Denoting the initial data rate as $R=n / N$, where $n$ and $N$ are the information bits and code bits, respectively, the per-user throughput can be expressed as

$$
\begin{aligned}
\eta & \doteq \frac{E\{\text { successfully decoded bits }\}}{E\{\text { number of channel uses }\}} \\
& =\frac{n p_{c}(M+1, R)}{\sum_{m=1}^{M+1} m N \operatorname{Pr}\left(A_{m}\right)} \\
& =\frac{R p_{c}(M+1, R)}{\sum_{m=1}^{M+1} m \operatorname{Pr}\left(A_{m}\right)}
\end{aligned}
$$

Here, the first equality comes from the renewal-reward theorem [12]-[14] and $\operatorname{Pr}\left(A_{m}\right)$ denotes the probability that the (re)transmissions end at the $m$-th round (and not before). The probability $\operatorname{Pr}\left(A_{m}\right)$ is given by

$$
\begin{aligned}
\operatorname{Pr}\left(A_{m}\right)= & \sum_{i=1}^{K} a_{i} \operatorname{Pr}\left(\log \left(1+(m-1) \operatorname{SIR}_{i}\right)<R\right. \\
& \left.\leq \log \left(1+m \operatorname{SIR}_{i}\right)\right) \\
= & p_{c}(m, R)-p_{c}(m-1, R),
\end{aligned}
$$
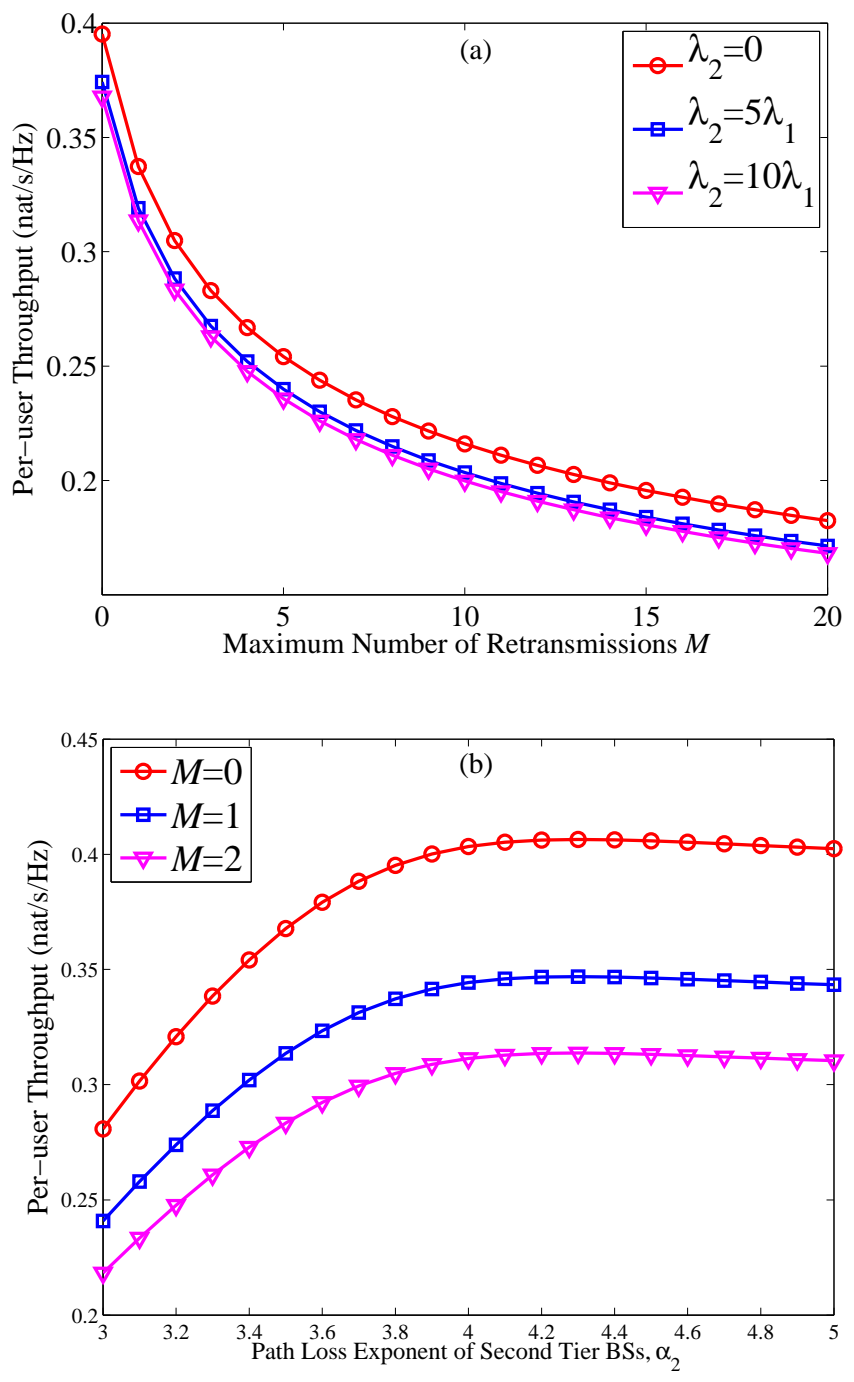

Figure 4. (a) Per-user throughput versus the maximum number of retransmissions $M$ in a 2-tier HetNet. The initial transmission rate of all BSs is set to $R=0.8149 \mathrm{nat} / \mathrm{s} / \mathrm{Hz}$. For tier- $1 \mathrm{BSs}$, the path loss exponent, the BS density and the transmit power are set to $\alpha_{1}=3.8, \lambda_{1}=\frac{1}{\pi 500^{2}}$ and $P_{1}=53 \mathrm{dBm}$, respectively. For tier-2 BSs, these parameters are considered as $\alpha_{2}=3.5, \lambda_{2}=0,5 \lambda_{1}, 10 \lambda_{1}$ and $P_{2}=33 \mathrm{dBm}$, respectively. (b) Peruser throughput versus the path loss exponent of second tier BSs in a 2-tier HetNet. All considered parameters are the same as before, except that we consider varying $\alpha_{2}$ and a fixed $\lambda_{2}=10 \lambda_{1}$.

for $1 \leq m \leq M$, and

$$
\operatorname{Pr}\left(A_{M+1}\right)=1-\sum_{m=1}^{M} P\left(A_{m}\right)=1-p_{c}(M, R),
$$

for $m=M+1$.

Hence, the per-user throughput in a $K$-tier HetNet under HARQ with the maximum number of retransmissions $M$ is given by

$$
\begin{aligned}
\eta= & R p_{c}(M+1, R) \times \\
& \left\{\sum_{m=1}^{M} m\left[p_{c}(m, R)-p_{c}(m-1, R)\right]+\right.
\end{aligned}
$$




$$
\left.(M+1)\left[1-p_{c}(M, R)\right]\right\}^{-1},
$$

which, using (9), can be evaluated numerically or analytically.

In Figure 3, we calculate the per-user throughput based on (16). As it is seen, although the QoS coverage probability improves by using HARQ (Figs. 1-2), for the considered parameter settings of the figure, the per-user throughput decreases with $M$. The explanation is that, while the QoS coverage is improved by retransmitting the same data, the expected number of channel uses also increases, leading to a loss in the throughput. However, the throughput loss is negligible particularly at low and high transmission rates. Moreover, the figure indicates that there exists an optimal initial transmission rate maximizing the per-user throughput and the optimal initial transmission rate maximizing the throughput is (almost) insensitive to the maximum number of retransmissions. For low initial transmission rate, although the QoS coverage probability is high, the per-user throughput is bad and vice versa. Thus, there is a trade-off between the initial transmission rate and the QoS coverage probability, in terms of the per-user throughput. Finding the analytical expression for the optimal initial transmission rate maximizing the per-user throughput is left for future work.

Figure 4(a) shows the effects of the maximum number of retransmissions and the second tier BS density on the per-user throughput. Again, the per-user throughput decreases with $M$ for all $\lambda_{2}$. However, the change of the per-user throughput is small due to further increasing the second tier BS density. Therefore, adding more small cell BSs does not affect the per-user throughput significantly, but allow more users to be served, thus increasing the overall throughput.

The per-user throughput under different path loss exponents of second tier BSs, i.e., $\alpha_{2}$, is illustrated in Fig. 4(b). For all values of $\alpha_{2}$, there is always a loss in the throughput by increasing the maximum number of retransmissions. Also, note that the per-user throughput does not increase monotonically with $\alpha_{2}$ for all $M$. This is because the larger the $\alpha_{2}$, the less the interference from the second tier BSs. When $\alpha_{2}$ is large, the per-user throughput only depends on the parameters of the first tier BSs.

\section{CONCLusions}

Implementing hybrid automatic repeat request (HARQ), this paper studies the QoS coverage probability and the peruser throughput of the heterogeneous networks (HetNets) modelled by a homogeneous Poisson Point process (PPP). We derived closed-form expressions for the network QoS coverage probability and the throughput. Also, we showed that the QoS coverage is improved substantially as the maximum number of retransmissions increases. However, depending on the channel condition, the implementation of HARQ may lead to slight throughput reduction. Furthermore, we demonstrated that the QoS coverage probability and the per-user throughput are almost invariant to the density of small cells. In addition, the per-user throughput increases with the small cell path loss exponent. Future works include the energy efficiency in
HetNets considering the power consumption of the different tiers, as well as implementation of adaptive modulation and coding in conjunction with HARQ.

\section{ACKNOWLEDGEMENTS}

This work was supported in part by the Swedish Governmental Agency for Innovation Systems (VINNOVA) within the VINN Excellence Center Chase, and the Swedish Research Council VR under the project 621-2009-4555 Dynamic Multipoint Wireless Transmission.

\section{REFERENCES}

[1] A. Ghosh, N. Mangalvedhe, R. Ratasuk, B. Mondal, M. Cudak, E. Visotsky, T. A. Thomas, J. G. Andrews, P. Xia, H. S. Jo, H. S. Dhillon, and T. D. Novlan, "Heterogeneous cellular networks: From theory to practice," IEEE Commun. Mag., vol. 50, no. 6, pp. 54-64, June 2012.

[2] J. Andrews, H. Claussen, M. Dohler, S. Rangan, and M. Reed, "Femtocells: Past, present, and future," IEEE J. Sel. Areas Commun., vol. 30, no. 3, pp. 497-508, 2012

[3] V. Chandrasekhar, J. Andrews, and A. Gatherer, "Femtocell networks: a survey," IEEE Commun. Mag., vol. 46, no. 9, pp. 59-67, Sept. 2008.

[4] A. Wyner, "Shannon-theoretic approach to a Gaussian cellular multipleaccess channel," IEEE Trans. Inf. Theory, vol. 40, no. 6, pp. 1713-1727, Nov. 1994.

[5] M. Haenggi, J. G. Andrews, F. Baccelli, O. Dousse, and M. Franceschetti, "Stochastic geometry and random graphs for the analysis and design of wireless networks," IEEE J. Sel. Areas Commun., vol. 27, no. 7, pp. 1029-1046, Sept. 2009.

[6] M. Haenggi, Stochastic geometry for wireless networks. Cambridge : Cambridge University Press, 2013.

[7] J. G. Andrews, F. Baccelli, and R. K. Ganti, "A tractable approach to coverage and rate in cellular networks," IEEE Trans. Commun., vol. 59, no. 11, pp. 3122-3134, Nov. 2011.

[8] H. S. Dhillon, R. K. Ganti, F. Baccelli, and J. G. Andrews, "Modeling and analysis of k-tier downlink heterogeneous cellular networks," IEEE J. Sel. Areas Commun., vol. 30, no. 3, pp. 550-560, Apr. 2012.

[9] H.-S. Jo, Y. J. Sang, P. Xia, and J. G. Andrews, "Heterogeneous cellular networks with flexible cell association: A comprehensive downlink SINR analysis," IEEE Trans. Commun., vol. 11, no. 10, pp. 3484-3495, Oct. 2012.

[10] X. Zhang and M. Haenggi, "A stochastic geometry analysis of inter-cell interference coordination and intra-cell diversity," IEEE Trans. Wireless Commun., 2013.

[11] H. Dhillon, M. Kountouris, and J. Andrews, "Downlink MIMO HetNets: Modeling, ordering results and performance analysis," IEEE Trans. Wireless Commun., vol. 12, no. 10, pp. 5208-5222, Oct. 2013.

[12] B. Makki and T. Eriksson, "On the average rate of HARQ-based quasistatic spectrum sharing networks," IEEE Trans. Wireless Commun., vol. 11 , no. 1 , pp. 65-77, Jan. 2012

[13] - "On the average rate of quasi-static fading channels with ARQ and CSI feedback," IEEE Commun. Lett., vol. 14, no. 9, pp. 806-808, Sept. 2010.

[14] C. Shen, T. Liu, and M. Fitz, "On the average rate performance of hybrid-ARQ in quasi-static fading channels," IEEE Trans. Commun., vol. 57, no. 11, pp. 3339-3352, Nov. 2009.

[15] F. Peng, J. Zhang, and W. Ryan, "Adaptive modulation and coding for IEEE 802.11n," in Wireless Communications and Networking Conference, 2007.WCNC 2007. IEEE, Mar. 2007, pp. 656-661.

[16] "Mobile WiMAX_Part ii: A comparative analysis," WiMAX Forum, May 2006.

[17] 3GPP TS 25.321 version 12.1.0 Release 12; Medium Access Control (MAC) protocol specification, Sept. 2014.

[18] B. Makki, T. Svensson, and M. Zorzi, "Finite block-length analysis of the incremental redundancy HARQ," IEEE Commun. Lett., 2014, DOI 10.1109/LWC.2014.2353059.

[19] A. Chelli and M. Alouini, "Performance of hybrid-ARQ with incremental redundancy over relay channels," in Globecom Workshops (GC Wkshps), 2012 IEEE, Dec. 2012, pp. 116-121. 